\title{
Real-time chemical imaging of working catalytic membrane reactors
}

$\underline{\text { Antonios Vamvakeros }}^{1}$, Simon D. M. Jacques ${ }^{2}$, Vesna Middelkoop ${ }^{3}$, Marco Di Michiel ${ }^{4}$, Andrew M. Beale ${ }^{1}$ ${ }^{1}$ Department Of Chemistry, University College London, London, United Kingdom, ${ }^{2}$ School of Materials, University of Manchester, Manchester, United Kingdom, ${ }^{3}$ Flemish Institute for Technological Research, VITO NV, Mol, Belgium, ${ }^{4}$ ESRF, Grenoble, France

E-mail: antonios.vamvakeros.12@ucl.ac.uk

The imaging of catalysts and other functional materials under reaction conditions has advanced significantly in recent years. The combination of the computed tomography approach with X-ray diffraction (XRD-CT) enables local chemical and physical state information to be extracted from within the interiors of intact materials which can be, by accident or design, commonly inhomogeneous. The spatially-resolved signals obtained can reveal information that would otherwise be lost in bulk measurement. Studying intact materials rather than idealised powders allows for behaviour under industrially relevant conditions to be observed. We will show here how XRD-CT has been used to track, for the first time, the evolving solidstate chemistry taking place inside a working catalytic membrane reactor used for the oxidative coupling of methane [1]. Furthermore, recent technical advancements in the XRD-CT technique will also be discussed. More specifically, a new data filtering strategy to remove/suppress artefacts generated in XRD-CT data due to large crystallites present in the sample [2] and a new data collection strategy, introduced as interlaced XRD-CT, which bridges the gap between spatial and temporal resolution of an XRD-CT scan [3], will be presented.

[1] A. Vamvakeros et al., Chemical Communications, 51, 12752-12755.

[2] A. Vamvakeros et al., Journal of Applied Crystallography, 48, 1943-1955.

[3] A. Vamvakeros et al., Journal of Applied Crystallography, 49, 485-496.

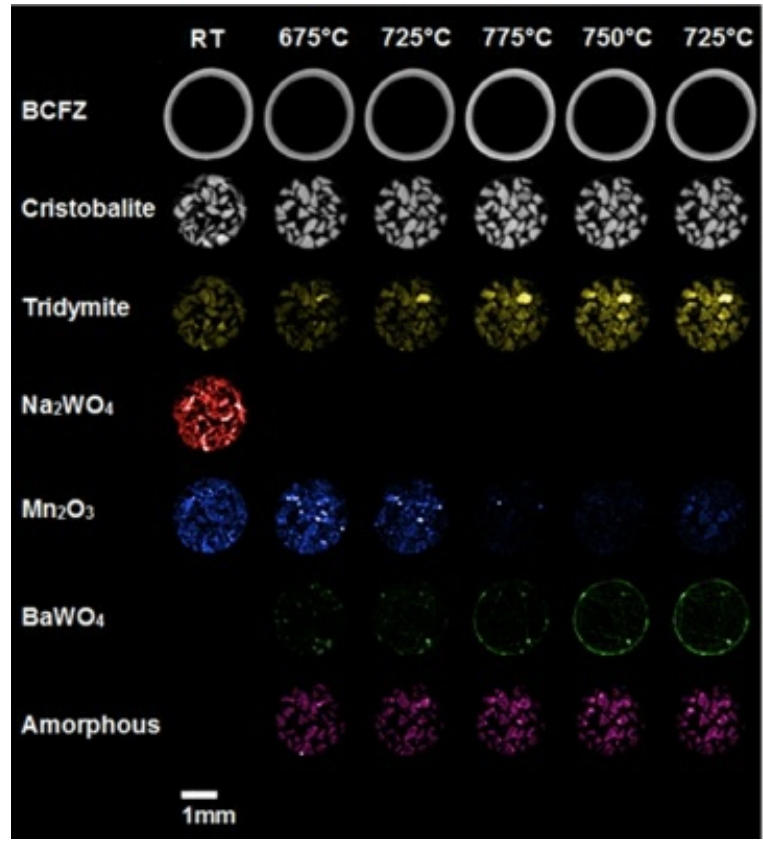

Filters for XRD-CT data
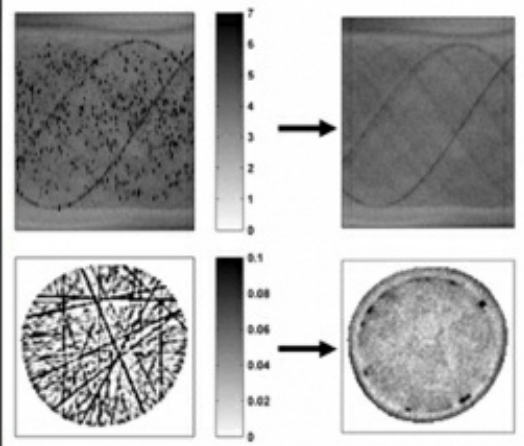

Interlaced XRD-CT method

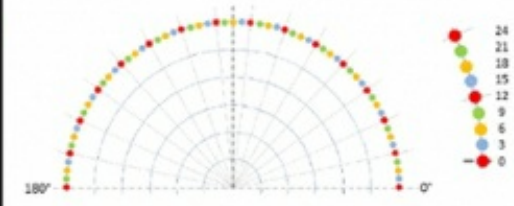

Keywords: heterogeneous catalysis, functional materials, chemical tomography 